\title{
Ethylene in Controlled Environments
}

\author{
sponsored by the
}

Growth Chambers and Controlled Environment Working Group

held at the

ASHS-98

Sacramento, California

22-25 July 2001 